\title{
COVID-19 EN MÉXICO: ASOCIACIÓN ESPACIAL DE CARA A LA FASE TRES
}

\section{COVID-19 NO MÉXICO: PARCERIA ESPACIAL PARA A TERCEIRA FASE}

\author{
Marcela Virginia Santana Juárez \\ Doctora en Geografía, Facultad de Geografía \\ Universidad Autónoma del Estado de México \\ mvsantana7@hotmail.com
}

Giovanna Santana Castañeda Doctora en Geografía, Facultad de Geografía Universidad Autónoma del Estado de México gsantanac@uaemex.mx

Christian Sánchez Carillo Mtro. En Análisis Espacial y Geoinformática, Facultad de Geografía Universidad Autónoma del Estado de México christian sc22@hotmail.com

Ronald Sánchez Carrillo Lic. En Geografía, Facultad de Geografía Universidad Autónoma del Estado de México ronald93 fg@hotmail.com

Roque Ortega Alcántara

Mtro. En Análisis Espacial y Geoinformática Instituto Nacional de Ecología y Cambio Climático biclo.kona@hotmail.com

\section{RESUMEN}

El propósito de este trabajo es presentar el comportamiento espacio temporal de la pandemia del COVID-19 en México, con un abordaje desde el análisis espacial, del 01 al 26 de abril de 2020, que incluye parte de la fase 2 y parte de la fase 3 de la pandemia. El método utilizado es el Índice de Moran y su gráfico de dispersión y se utilizan los gráficos de LISA (Local Indicator of Spatial Association) para analizar la dependencia espacial local, de los casos por COVID-19. Los resultados señalan que durante la fase 2 de la pandemia se caracteriza principalmente por los contagios comunitarios, en las principales zonas metropolitanas. En la tercera etapa el contagio es local, expandiéndose en forma exponencial aproximadamente 800 casos por día. En estados del centro de México, Baja California (limítrofe con los Estados Unidos), Tabasco y Quintana Roo, que registran los mayores casos. La principales conclusiones derivadas del análisis espacial es que existen grupos o clusters de municipios que son focos rojos porque registran el mayor número de casos, rodeados por municipios con valores altos; municipios con valores altos rodeados por municipios con valores bajos; municipios con valores bajos acorralados por municipios con valores altos; así como municipios con valores bajos rodeados por municipios bajos, que dependiendo de ello las políticas y medidas de mitigación deben ser diferenciadas y rigurosas, sin descuidar algún municipio.

Palabras clave: COVID-19. autocorrelación espacial. comportamiento espacial.

\section{RESUMO}

O objetivo deste artigo é apresentar o comportamento espacial-temporal da pandemia da COVID-19 no México, com uma abordagem da análise espacial, de 1 a 26 de Abril de 2020, que inclui parte da fase 2 e parte da fase 3 da pandemia. O método utilizado é o índice Moran e a sua parcela de dispersão e os gráficos LISA (Local Indicator of Spatial Association) são utilizados para analisar a dependência espacial local dos casos pela COVID-19. Os resultados indicam que, durante a fase 2 da pandemia, esta se caracteriza principalmente pelo contágio da

Recebido em: 29/04/2020

Aceito para publicação em: 02/06/2020. 


\begin{abstract}
comunidade, nas principais áreas metropolitanas. Na terceira fase, o contágio é local, expandindo-se exponencialmente para cerca de 800 casos por dia. Nos Estados centrais mexicanos, Baja California (que faz fronteira com os Estados Unidos da América), Tabasco y Quintana Roo, que registam o maior número de casos. As principais conclusões da análise espacial são que existem grupos ou aglomerados de municípios que são pontos críticos porque registam o maior número de casos, rodeados por municípios com valores elevados; municípios com valores elevados rodeados por municípios com valores baixos; municípios com valores baixos encurralados por municípios com valores elevados; bem como os municípios com valores baixos rodeados por baixos, que, consoante o caso, as políticas e medidas de atenuação devem ser diferenciadas e rigorosas, sem descurar nenhum municipio.
\end{abstract}

Palavras-chave: COVID-19. autocorrelação espacial. comportamento espacial.

\title{
PRESENTACIÓN
}

La pandemia del COVID-19 es un evento de gran magnitud que se presenta de forma diferenciada en el territorio internacional y la difusión se registró muy rápidamente a través de un mundo globalizado, por medio de la movilidad, por vuelos internacionales principalmente que rápido se propago de China a Europa y de Europa hacía América, con vuelos hasta de diez horas atravesando los océanos, pese a encontrarse hasta el otro lado de China.

Las epidemias no son eventos nuevos, en la historia del hombre siempre ha habido propagación de virus, en el caso de la Nueva España se desarrollaron epidemias devastadoras como la viruela, de tifo y el sarampión, entre otros.

En muchas de estas epidemias el hombre implementado como política principal las cuarentenas, las cuales se ha demostrado que son eficaces para cortar cadenas de contagios, en la actualidad hay cuarentenas parciales y restrictivas diferenciándose por su rigidez en la implementación, por lo que es importante que las políticas que se implementen algunas sean generales y otras específicas, pero principalmente que se diferencien en el territorio acorde a cada fase en el desarrollo de la epidemia.

Este trabajo se estructura en tres apartados que incluye la metodología para desarrollar las técnicas de autocorrelación espacial global y local, el contexto de la pandemia por COVID-19 en México así como sus fases; el análisis de la autocorrelación espacial y conclusiones.

\section{MÉTODOS}

El método del Índice de Moran y su gráfico de dispersión permiten analizar la dependencia o autocorrelación espacial desde un punto de vista global, pero además de ello, se necesitan detectar las zonas "calientes" o "frías" que se desvían de la tendencia general de una variable o de la presencia o no de concentraciones espaciales, para ello se utilizan los gráficos de LISA (Local Indicator of Spatial Asociation) para analizar la dependencia espacial local.

Esta técnica permite localizar los valores significativos en determinados indicadores estadísticos de asociación espacial local (Unwin, 1996), y se basa en el estadístico I de Moran de asociación local. Se trata de un estadístico que adquiere un valor diferente para todas y cada una de las unidades espaciales.

El diagrama de dispersión de Moran está representado por dos ejes x e y, en el eje $X$ se muestra la variable que se va a analizar y en el eje $Y$ se representa el retardo espacial de dicha variable, entendiendo al retardo espacial como el promedio ponderado de los vecinos, dependiendo de cuál matriz de pesos se utilice.

En términos generales, la autocorrelación puede ser positiva o negativa, cuando es positiva la presencia de alguna característica en una unidad espacial, llámese municipio, conduce al aumento de esa misma característica hacía el resto de los municipios que la rodean, favoreciendo la concentración de este, por ejemplo, en un tablero de ajedrez se colocan todas las casillas blancas en la primera mitad y en la segunda todas las casillas negras, esto se expresa en la figura 1, a ello se le llama correlación perfecta.

El caso contrario es cuando la presencia de alguna característica en algún municipio impide o dificulta la aparición de regiones vecinas a ella, es decir, que las unidades espaciales vecinas tengan valores 
netamente diferentes entre ellas, tal como lo muestra la distribución de las casillas blancas y negras en un tablero de ajedrez, esto se puede ver gráficamente en la figura 2, lo que significa una dispersión perfecta y de forma matemática en la ecuación 1.

Figura 1 - Correlación perfecta

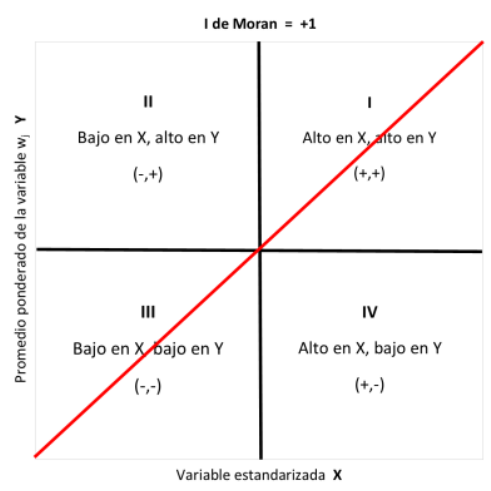

Figura 2 - Dispersión perfecta

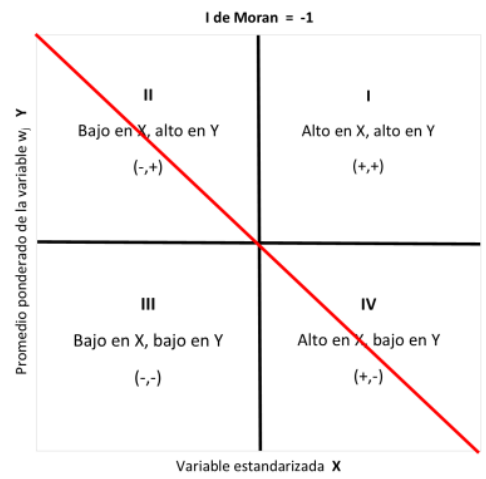

Fuente: Santana y Aguilar, (2019)

El resultado oscila entre -1 que indica dispersión perfecta y +1 que indica correlación perfecta y el valor de cero indica un patrón espacial aleatorio, esto significa que, si los valores similares tienden a agruparse espacialmente, por ejemplo, los valores altos cerca de otros valores altos o valores bajos cerca de otros valores bajos, el Índice de Moran será positivo, sin embargo, cuando hay valores altos rodeados de valores bajos o viceversa, el Índice será negativo (ecuación 2)

$$
I=\frac{n \Sigma_{i}\left(x_{i}-x\right) \Sigma_{j} w_{j}\left(x_{j}-x t\right)}{\left(\Sigma_{i} \Sigma_{j} w_{j}\right) \Sigma_{i}\left(x_{i}-x\right)^{2}}
$$

Ecuación 1. I de Moran

Fuente: Elaborado con base en Santana y Aguilar, 2019

$$
I_{i}=\frac{x_{i}-\bar{R}}{s_{i}^{2}} \sum_{j=1_{j} j \neq i}^{n} w_{i j}\left(x_{i}-\bar{X}\right)
$$

Ecuación 2. Local del I de Moran

Fuente: Elaborado sobre con base en Mitchell, A 2005.

Dónde $n$ es el número de unidades espaciales,

$x_{i}$ es el valor de la variable en la unidad espacial i,

$x_{\tilde{j}}$ es el valor de la variable en la otra localización,

$\bar{x}$ es la media de la variable y

$w_{j}$ es la ponderación que indica la relación de contigüidad entre las unidades espaciales $i-j ;$ Si $i-j$

comparten límites $W_{i j}=1$ si no $W_{i j}=0$

Este método se base en la primera ley de la Geografía propuesta por Tobler en 1970, que señala que todo está relacionado entre sí, sin embargo, las cosas que se encuentran más próximas en el espacio tienen una mayor relación que entre las que se encuentran distantes.

En lo que respecta a la enfermedad del COVID-19, está ley se observa con mayor claridad en la segunda y tercera etapa del contagio, debido a que un gran número de contagios implica una gran preocupación, debido a la naturaleza propia de esta enfermedad. 


\section{CONTEXTO GENERAL DE LA PANDEMIA DEL COVID-19 EN MÉXICO}

De acuerdo con la Organización Mundial de la Salud (OMS), la Organización Panamericana de la Salud (OPS) y el Gobierno Federal de México, mediante la Subdirección de Prevención y Promoción de la Salud, los hechos más relevantes sobre la pandemia del COVID-19 en México son los que se presentan a continuación.

El 10 de diciembre de 2019, en Wuhan, China, inició la pandemia del COVID-19, causada por el virus SARS-CoV-2, 20 días después el Dr. Wenliang alerta sobre los primeros casos en esa misma ciudad, para el 13 de enero de 2020, se confirma el primer caso fuera de China, localizado en Tailandia y el 30 de enero de 2020 la OMS declara la epidemia de COVID-19 como una Emergencia de Salud Pública de Importancia Internacional (ESPII).

En un mundo globalizado a través de la movilidad internacional la pandemia se ha expandido de China a Italia, España y a los Estados Unidos, de manera acelerada, por ejemplo el 21 de enero se reporta el primer caso en el continente americano de un hombre que había viajado de China a Estados Unidos; mientras que el 31 de enero se reportan los primeros casos en Italia cuando dos turistas chinos dan positivo en Roma; para el caso de México se reportaron tres casos el 28 de febrero de un italiano de 35 años de edad, residente de la Ciudad de México, el segundo caso de un ciudadano del Estado de Hidalgo que se encontraba en el Estado de Sinaloa y el tercer caso de la Ciudad de México, todos ellos procedentes de Italia.

El 11 de marzo el director de la OMS declaró la actual epidemia de COVID-19 como pandemia, en el caso de México para el 24 de marzo suman 405 casos confirmados y 5 muertes, es en esta fecha que se registran los primeros contagios locales, que aumentan rápidamente, debido a ello el Gobierno Federal decretó el inicio de la fase 2 de esta pandemia: Para el 30 de marzo, se declaró emergencia sanitaria por causa de fuerza mayor y para el 31 de marzo ya habían 1,215 casos confirmados y 29 defunciones.

\section{COVID-19 EN MÉXICO DEL 01 AL 26 DEL MES DE ABRIL, 2020}

Para México, el mes de abril inicia con un acumulado de 1378 infectados oficiales y 37 defunciones, para este día se posiciona en el lugar 450 a nivel internacional. Los países con la mayor cantidad de infecciones por el COVID- 19, son Estados Unidos con 214,482, seguido por Italia y España con 110,574 y 104,116 respectivamente.

El 2 de abril, la cantidad de casos confirmados se incrementó a 1510, y 50 defunciones, los cuales se registraron en áreas urbanas, principalmente como en la Ciudad de México, los Estados de México y Jalisco, en menor proporción en los Estados fronterizos de Chihuahua, Coahuila y Nuevo León, así como Puebla. Hasta el momento, la tendencia de aumento diario de casos había mantenido un promedio de 150.

Un dato relevante es que para el 16 de abril se registraron 100 niños contagiados y para el 19 de abril da inicio un comportamiento activo de la pandemia.

Aunque cada día han aumentado la cantidad de personas que dan positivo a esta enfermedad, el 24 de abril se registró la mayor cantidad de casos nuevos con 1239, un total de casos acumulados de 12,872 y 1,221 muertes. La tendencia diaria de casos confirmados ha aumentado a más de 800 . Las mayores cantidades de casos, se presentaron en la Ciudad de México, Estado de México, Baja California, Sinaloa, Tabasco y Quintana Roo.

A nivel internacional, el 26 de abril se registraron 2'804,796 de casos y 193,710 muertes, abanderando el primer lugar Estados Unidos con 899,281 casos, continuando España con 219,764 e Italia con 195,351.

Para el 28 de abril, la OPS confirmó que México ocupa el quinto lugar de casos reportados de coronavirus en América. Estados Unidos, Brasil, Canadá, y Ecuador, son los cuatro que le superan (INFOBAE, 2020)

En la tabla 1 y 2 , y las figuras 3 y 4 , muestran el número de casos nuevos, los casos acumulados y las defunciones de lo que va en el mes de abril hasta la publicación del presente artículo. 

Christian Sánchez Carillo Ronald Sánchez Carrillo Roque Ortega Alcántara

Tabla 1 - México: COVID 19 por estados, 26 de abril de 2020

\begin{tabular}{|c|c|c|c|}
\hline Estado & $\begin{array}{c}\text { Casos confirmados al } \\
26 \text { de abril 2020, por } \\
\text { COVID-19 }\end{array}$ & $\begin{array}{l}\text { Total de muertes al } 26 \text { de abril } \\
\text { de } 2020 \text {, por COVID-19 }\end{array}$ & $\begin{array}{l}\text { Población total al } \\
2020\end{array}$ \\
\hline Aguascalientes & 148 & 2 & 1.425 .105 \\
\hline Baja California & 1271 & 162 & 3.606 .940 \\
\hline Baja California Sur & 284 & 12 & 796.398 \\
\hline Campeche & 69 & 14 & 992.306 \\
\hline Coahuila & 348 & 33 & 3.197 .188 \\
\hline Colima & 19 & 2 & 778.989 \\
\hline Chiapas & 122 & 6 & 5.688 .998 \\
\hline Chihuahua & 258 & 58 & 3.783 .680 \\
\hline Ciudad de México & 3966 & 309 & 9.025 .363 \\
\hline Durango & 44 & 6 & 1.861 .051 \\
\hline Guanajuato & 206 & 20 & 6.201 .449 \\
\hline Guerrero & 200 & 29 & 3.650 .850 \\
\hline Hidalgo & 182 & 18 & 3.068 .696 \\
\hline Jalisco & 282 & 25 & 8.368 .602 \\
\hline México & 2355 & 116 & 17.338 .220 \\
\hline Michoacán & 204 & 31 & 4.808 .791 \\
\hline Morelos & 190 & 27 & 2.033 .373 \\
\hline Nayarit & 63 & 7 & 1.279 .671 \\
\hline Nuevo León & 276 & 9 & 5.571 .904 \\
\hline Oaxaca & 106 & 15 & 4.132 .318 \\
\hline Puebla & 511 & 65 & 6.573 .843 \\
\hline Querétaro & 111 & 9 & 2.259 .471 \\
\hline Quintana Roo & 604 & 79 & 1.704 .010 \\
\hline San Luis Potosí & 78 & 6 & 2.856 .171 \\
\hline Sinaloa & 747 & 105 & 3.143 .980 \\
\hline Sonora & 167 & 18 & 3.056 .397 \\
\hline Tabasco & 728 & 95 & 2.558 .349 \\
\hline Tamaulipas & 261 & 11 & 3.635 .833 \\
\hline Tlaxcala & 134 & 13 & 1.372 .108 \\
\hline Veracruz & 354 & 25 & 8.514 .724 \\
\hline Yucatán & 323 & 20 & 2.246 .505 \\
\hline Zacatecas & 66 & 4 & 1.660 .543 \\
\hline Total & 14677 & 1351 & 127.191 .826 \\
\hline
\end{tabular}

Fuente: Elaboración propia con base en Gobierno Federal. Secretaría de Salud (2020) y Censo de población y vivienda 2010 y la Encuesta intercensal 2015 INEGI. 
Tabla 2 - México. Evolución de la pandemia por el SARS-CoV-2 que causa la enfermedad COVID 19 , al 26 de abril, 2020

\begin{tabular}{|c|c|c|c|}
\hline Fecha & Nuevos casos & $\begin{array}{l}\text { Casos confirmados } \\
\text { (acumulados) }\end{array}$ & $\begin{array}{l}\text { Número de } \\
\text { defunciones } \\
\text { acumuladas }\end{array}$ \\
\hline $01 / 04 / 2020$ & 163 & 1378 & 37 \\
\hline $02 / 04 / 2020$ & 132 & 1510 & 50 \\
\hline $03 / 04 / 2020$ & 178 & 1688 & 60 \\
\hline $04 / 04 / 2020$ & 202 & 1890 & 79 \\
\hline $05 / 04 / 2020$ & 253 & 2143 & 94 \\
\hline $06 / 04 / 2020$ & 296 & 2439 & 125 \\
\hline $07 / 04 / 2020$ & 346 & 2785 & 141 \\
\hline $08 / 04 / 2020$ & 396 & 3181 & 174 \\
\hline $09 / 04 / 2020$ & 260 & 3441 & 194 \\
\hline $10 / 04 / 2020$ & 403 & 3844 & 233 \\
\hline $11 / 04 / 2020$ & 375 & 4219 & 273 \\
\hline $12 / 04 / 2020$ & 442 & 4661 & 296 \\
\hline $13 / 04 / 2020$ & 353 & 5014 & 332 \\
\hline $14 / 04 / 2020$ & 385 & 5399 & 406 \\
\hline $15 / 04 / 2020$ & 448 & 5847 & 449 \\
\hline $16 / 04 / 2020$ & 450 & 6297 & 486 \\
\hline $17 / 04 / 2020$ & 578 & 6875 & 546 \\
\hline $18 / 04 / 2020$ & 622 & 7497 & 650 \\
\hline $19 / 04 / 2020$ & 764 & 8261 & 686 \\
\hline $20 / 04 / 2020$ & 511 & 8772 & 712 \\
\hline $21 / 04 / 2020$ & 729 & 9501 & 857 \\
\hline $22 / 04 / 2020$ & 1043 & 10544 & 970 \\
\hline $23 / 04 / 2020$ & 1089 & 11633 & 1069 \\
\hline $24 / 04 / 2020$ & 1239 & 12872 & 1221 \\
\hline $25 / 04 / 2020$ & 970 & 13842 & 1305 \\
\hline $26 / 04 / 2020$ & 835 & 14677 & 1351 \\
\hline
\end{tabular}

Fuente: Elaboración propia con base en Gobierno Federal. Secretaría de Salud (2020). 
Figura 3 - México, pandemia por SARS COV-2 que causa la enfermedad COVID-19. Casos confirmados al 26 de abril de 2020

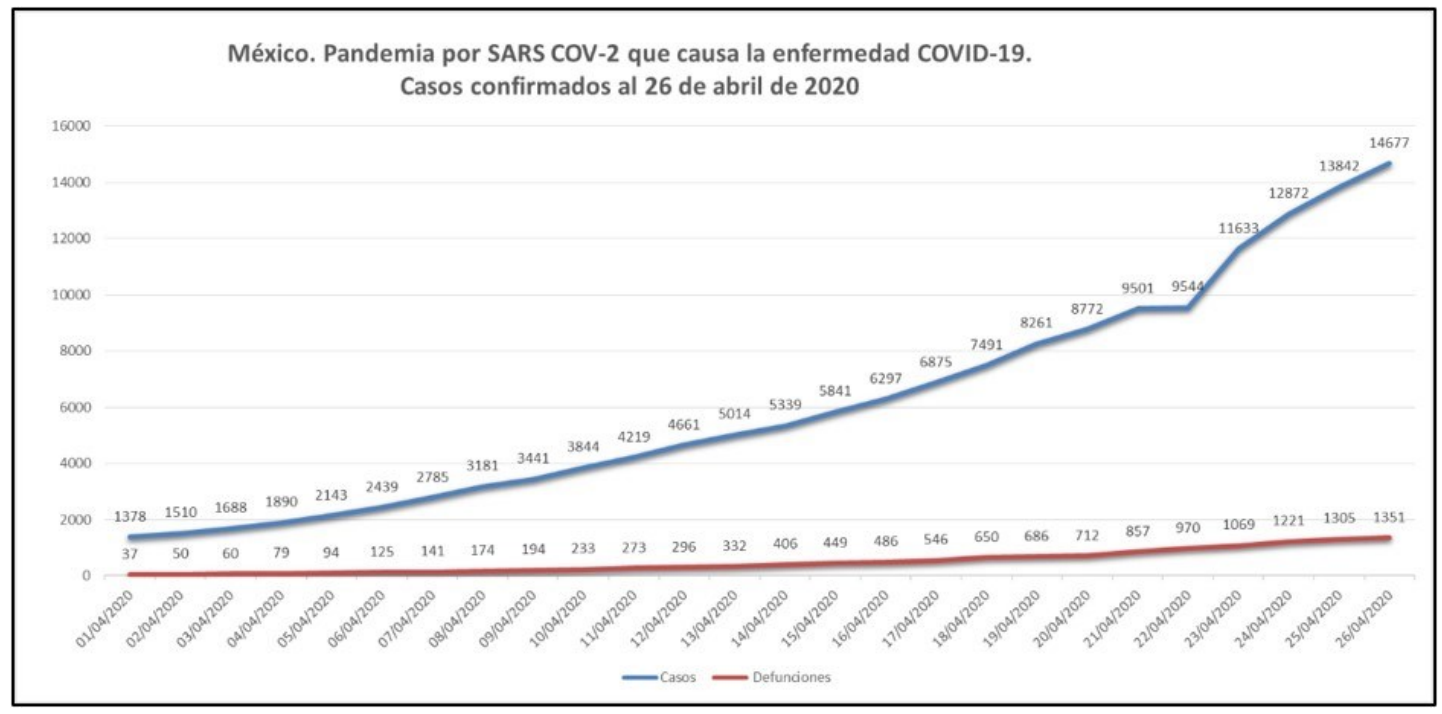

Fuente: Elaboración propia con base en Gobierno Federal. Secretaría de Salud (2020).

Figura 4 - México, distribución de casos confirmados y defunciones por COVID-19 a nivel municipal. 26 de abril de 2020

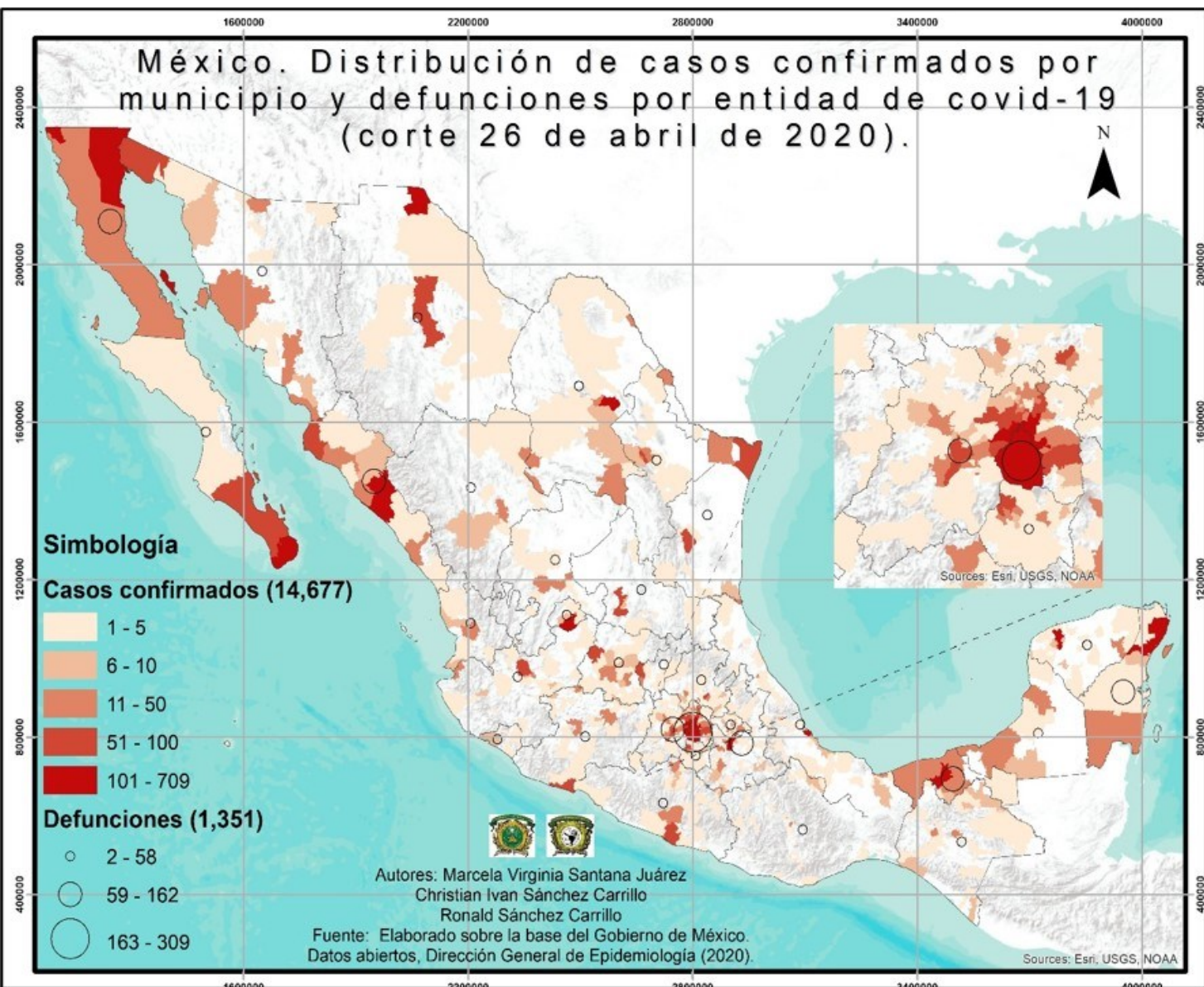




\section{FASES DE LA PANDEMIA DEL COVID-19 EN MÉXICO}

La primera fase de la pandemia inició el 28 de febrero de 2020 y duró hasta el 23 de marzo, esta etapa se caracteriza por la importación de casos del extranjero, principalmente de Estados Unidos, España, e Italia y existe un número limitado de personas infectadas con el virus. Las medidas de sanidad incluyen acciones de prevención de propagación con la jornada Nacional de Sana Distancia, así como la cuarentena permaneciendo en casa e inician los comunicados diarios por parte del Gobierno Federal.

La segunda fase de la pandemia comprende del 24 de marzo al 20 de abril de 2020, la cual se caracteriza por la transmisión comunitaria en la que no es posible conocer las redes de contagio, la expansión es acelerada y se concentra principalmente en la Ciudad de México y en los estados de, México, Baja California, Tabasco, Sinaloa, además de las zonas metropolitanas de Guadalajara y Monterrey (figuras 5 y 6 ).

En esta fase, el Gobierno de México, a través del Subsecretario de Prevención y Promoción de la salud, Hugo López Gatell, presentó el modelo centinela, este sistema de vigilancia calcula que la epidemia de COVID-19 es ocho veces mayor que lo reportado, esto permite vigilar el comportamiento espacial de la pandemia con estimaciones del sitio y la cantidad de personas con posibilidades de contraer el virus.

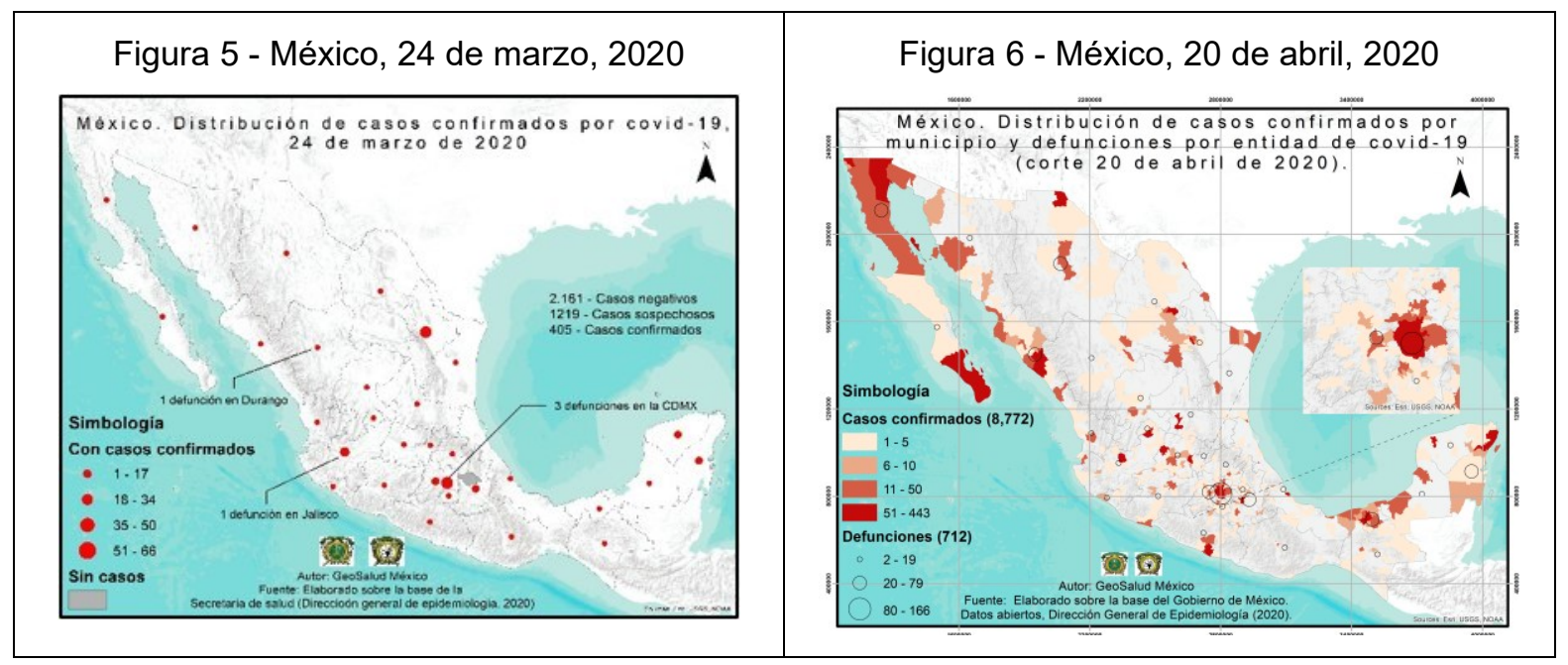

La tercera fase inició el 21 de abril de 2020, que se caracteriza por el aumento de los casos en forma exponencial y hospitalizaciones. La pandemia registra diferente velocidad de propagación en el territorio, dependiendo de los condicionantes socioespaciales como la distribución de la población, la densidad y la movilidad que inciden en que se expanda de manera diferenciada en el territorio a una velocidad desigual (Santana M, 2020).

Para el 21 de abril, se registraron 9,501 casos y 857 defunciones y según información del Gobierno federal, se prevé que entre el 08 y el 10 de mayo pudiera ser el auge o pico de la epidemia, el cual durará varias semanas. Esta fase está enfocada en los casos activos que suman 3,185 al 22 de abril, estos revelan las "cargas ocultas" que antes con los casos acumulados no se habían identificado (La Jornada, 2020a). Los casos activados hacen referencia a aquellas personas que iniciaron con síntomas 15 días antes, estos se presentan principalmente en la vertiente del Golfo de México, Veracruz, Tamaulipas y Tabasco.

Las cifras para el mismo día son 2,378 casos activos en la ciudad de México y el Estado de México, casi la mitad de los 4,972 contabilizados a escala nacional, en Tabasco la cantidad de casos activos era de 283 mientras que en Tamaulipas y Veracruz eran de 162 y 133 respectivamente, estos Estados empezaron repuntar en días pasados con una carga de enfermedad más elevada, respecto al resto de los Estados de México (La jornada, 2020b) 
El 25 de abril se implementó la Red de Infección Respiratoria Aguda Grave (Red IRAG), que son hospitales generales para atender personas con COVID-19, los cuales se concentran en mayor medida en el centro de México, y en donde se tienen las mayores cantidades de población y las mayores densidades de población

En el informe diario del 26 de abril sobre el COVID-19 por parte del Gobierno de México dieron a conocer que se cuenta con 618 hospitales notificantes y $536(87 \%)$ hospitales que notificaron, además se tienen 12,682 camas IRAG disponibles y 3,337 (20\%) ocupadas. A nivel nacional el $77 \%$ están disponibles y el $23 \%$ están ocupadas.

La ciudad de México cuenta con 2,100 camas disponibles, 1,112 están ocupadas y 988 (47\%) libres; el Estado de México cuenta con 2,137 camas de las cuales 859 están ocupadas y 1,278 (60\%) están disponibles; Baja California tiene 214 ocupadas y 296 disponibles (58\%). La Ciudad de México y el Estado de México registran las mayores cantidades de camas, porque registran las mayores cantidades de casos, población y densidad de población en México (figura 7).

Figura 7 - México, densidad de población y distribución de casos confirmados por COVID-19 al 26 de abril de 2020.

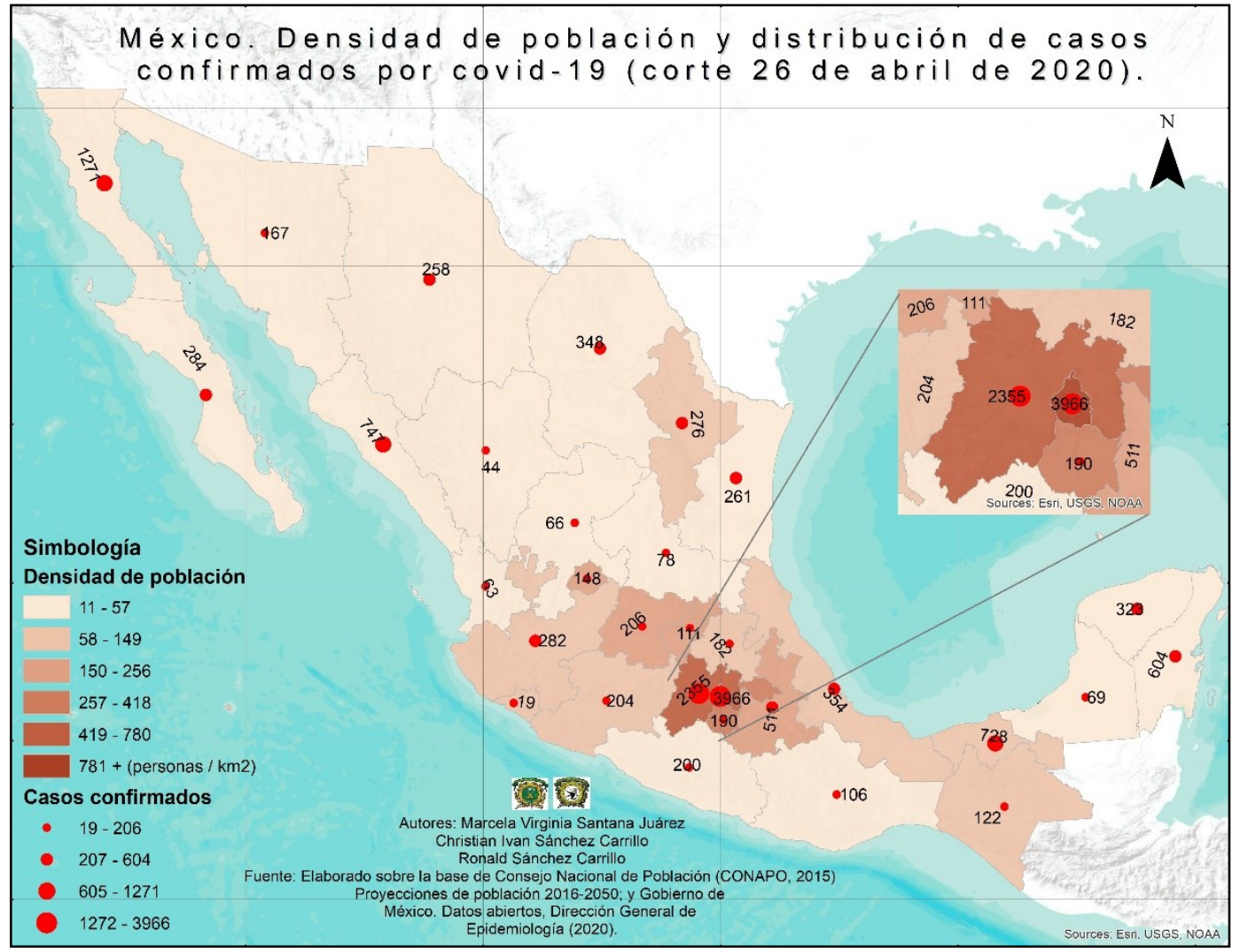

En relación a la ocupación de camas con ventilador: la ciudad de México reporta 310 ocupadas y 848 (73\%) disponibles; Estado de México 145 asignadas y 616 (81\%) desocupadas; y Baja California tiene 41 ocupadas y 199 (83\%) libres (La Jornada, 2020c).

El 26 de abril en México se registraron 14,677 casos y 1,351 defunciones, para esta fecha ocupa el lugar 25 con respecto al número de casos, se encuentra en la tercera etapa el número de casos y defunciones se incrementaron significativamente, en 26 días. (OMS, 2020)

En el comunicado técnico diario que realiza la Secretaria de Salud por parte de la Subdirección de prevención y promoción de la salud del 28 de abril, el Dr. Ricardo Cortez expuso el índice compuesto de movilidad comunitaria por Estado, cuyos insumos principales son Google y twitter en los que se DOI:http://dx.doi.org/10.14393/Hygeia0054317 Hygeia $\quad$ Edição Especial: Covid-19, Jun./2020 $\quad$ p.36 - 48, $\quad$ pág. 44 
muestran 28 Estados de la República mexicana que registran un índice de movilidad cerca del $50 \%$, mientras que cuatro que son Jalisco, Nuevo León, Oaxaca y Zacatecas tuvieron valores cercanos al $75 \%$, lo que indica que existe gran movilidad ciudadana, por lo que es importante continuar y reforzar las medidas de mitigación relacionadas a permanecer en casa y de igual manera el Gobierno de México insta a gobiernos estatales a restringir la movilidad.

La distribución de la población por grupos de edad, en México representa una gran fortaleza, la mediana de edad de los casos acumulados es 46 años, los grupos de población de 25 a 64 años de edad registraron los mayores números de casos, de los cuales el $58 \%$ son hombres y el $42 \%$ son mujeres. Las defunciones muestran en promedio 59 años de edad, se registraron principalmente entre los 45 y 74 años de éstos el $32 \%$ son mujeres, mientras el $68 \%$ son hombres.

Durante la fase 3 de la pandemia el Gobierno Federal exhorta a la población a permanecer bajo resguardo domiciliario de grupos de población en riesgo: niños, adultos mayores, personas con alguna enfermedad crónico degenerativa, mujeres embarazas, así como aquellas personas que realizan actividades clasificadas como no esenciales, a fin de cortar cadenas de contagio.

\section{AUTOCORRELACIÓN ESPACIAL DE LOS CASOS POR COVID-19 AL 26 DE ABRIL DE 2020}

El valor del índice de Moran de autocorrelación global va de -1 a +1 , y a medida que se acerque el valor restante a los números enteros será más fuerte el grado de autocorrelación espacial en la variable. Además de ello, la hipótesis nula para este método establece que las variables analizadas en los municipios se encuentran distribuidas de manera aleatoria, por lo tanto, la pregunta a responder es si existe un patrón de comportamiento espacial de concentración, dispersión o aleatoriedad dentro de los casos positivos por COVID-19 en México para el día 26 de abril. El resultado fue positivo de 0.367 , esto significa que el $37 \%$ de los municipios con valores altos se encuentran rodeados por municipios con altos valores, así como los valores bajos rodeados por valores bajos, y por lo tanto tienden a la concentración, esto se observa en la figura 8.

Figura 8 - COVID-19 en México. Índice de autocorrelación global de Moran, 26 de abril de 2020

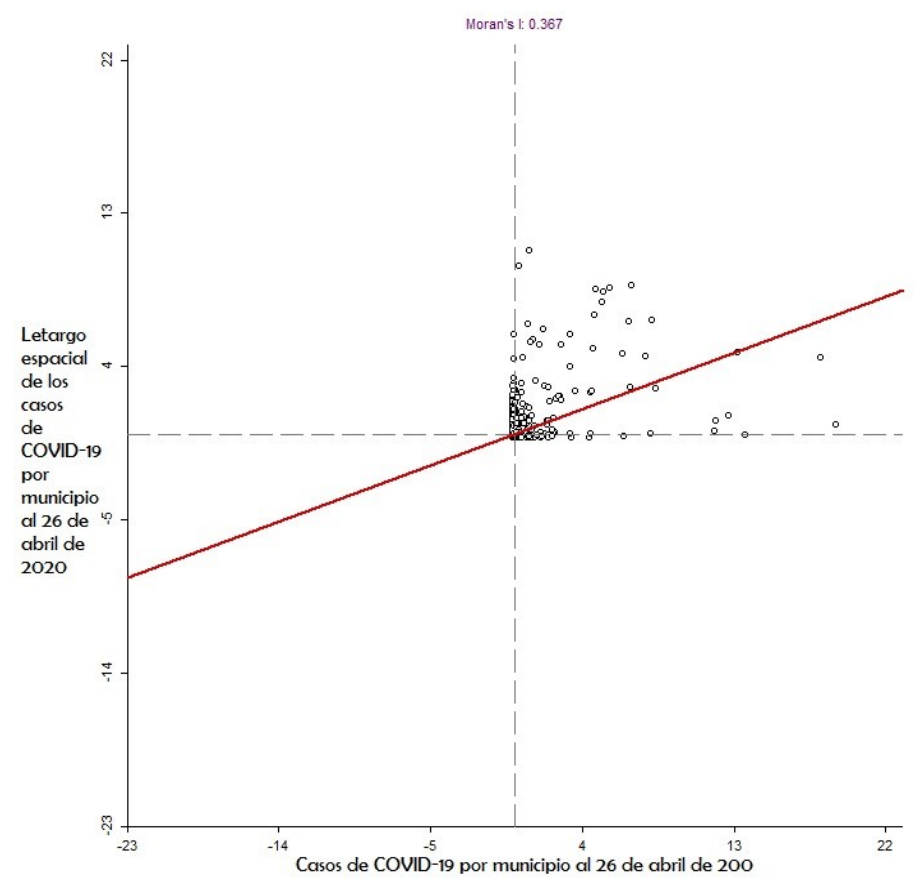

Fuente: Elaboración propia con base en el Gobierno de México, Dirección General de Epidemiología, 2020 y el software GEODA TM. 
El índice de autocorrelación espacial local de Moran permite analizar el comportamiento de los municipios y sus vecinos para identificar puntos calientes, puntos fríos y valores atípicos espaciales que son estadísticamente significativos.

La figura 9 identifica cuatro clústeres de municipios: en color rojo se observa el nivel de afectación crítico, en naranja el significativo y en amarillo el moderado y un grupo de municipios que tienen valores bajos rodeados de valores bajos en color verde con una afectación baja, se utilizó una matriz de pesos espaciales de contigüidad de reina y 999 permutaciones de Monte Carlo para generar estas métricas.

Figura 9 - COVID-19 en México. Índice de autocorrelación espacial local de Moran, 26 de abril de 2020

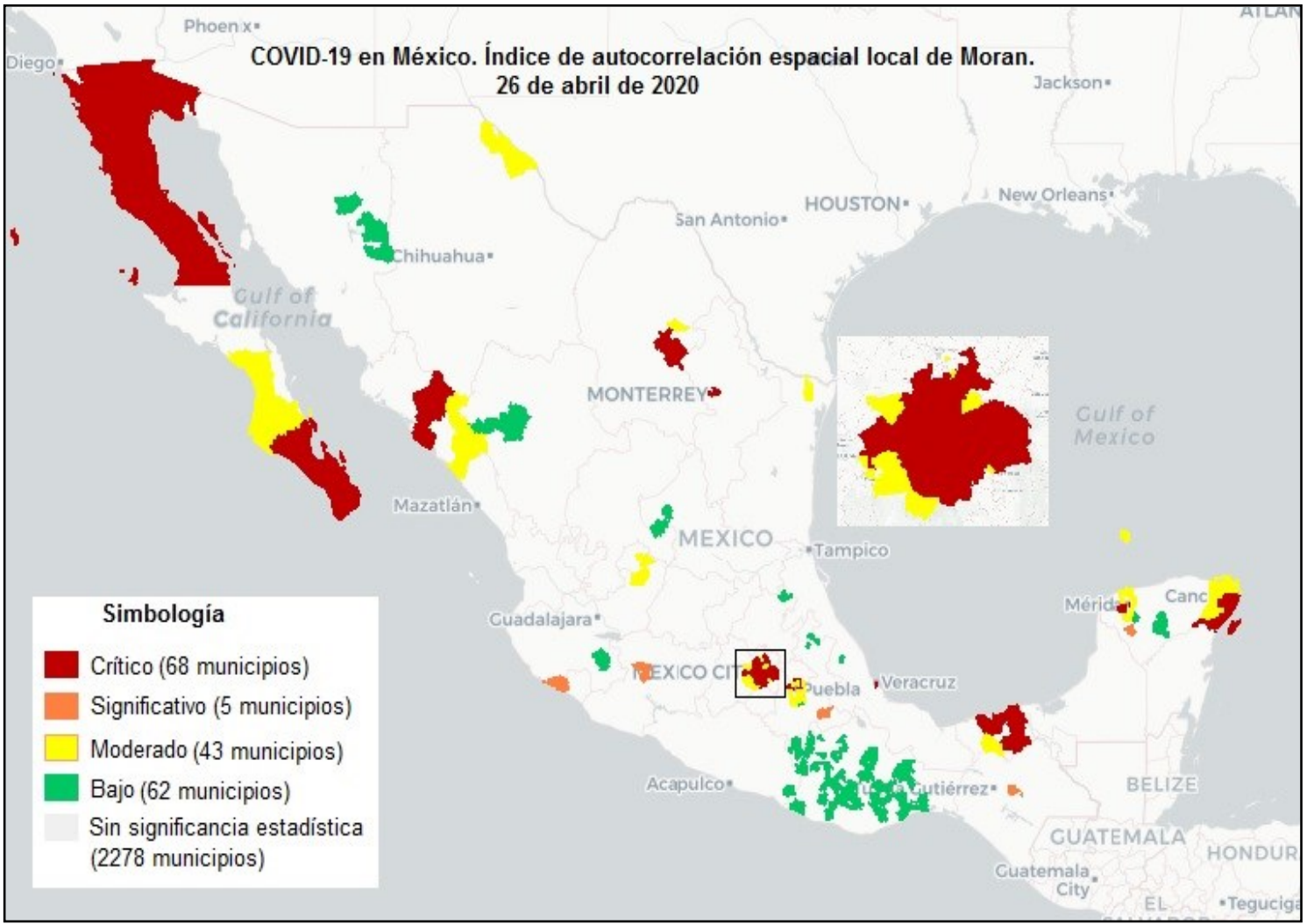

Fuente: Elaboración propia con base en el Gobierno de México, Dirección General de Epidemiología, 2020 y el software GEODA TM.

Los municipios, que en el mapa se identifican por los colores rojos, son los denominados puntos calientes y son de particular interés en el análisis geográfico debido a su grado de afectación, porque son municipios que cuentan con valores altos de casos positivos por covid-19, y que a su vez están rodeados de municipios con indicadores altos, Dentro de estos municipios se encuentran Iztapalapa, Gustavo A. Madero, Mexicali, Benito Juárez, Tlalpan, Nezahualcóyotl, Álvaro Obregón, Coyoacán, Ecatepec, Cuauhtémoc, entre otros; todos ellos son municipios eminentemente urbanos, con dinámicas de población y económica fuertes.

Los valores atípicos ocupan un lugar importante en el interés pandémico debido a que son municipios que tienen una gran cantidad de casos, pero se encuentran cerca de municipios con una cantidad baja; además existe otro conjunto de municipios que tienen bajos números de casos confirmados, pero que sus vecinos tienen valores altos, lo que destaca un riesgo emergente. 
En el mapa se destacan en color naranja cinco municipios que registran valores altos rodeados de valores bajos, entre ellos, se encuentran San Cristóbal de las Casas localizado en el Estado de Chiapas, Uruapan en Michoacán, Manzanillo en Colima, Tehuacán en Puebla y Ticul en Yucatán.

De color amarillo se enfatizan los municipios que registran valores bajos, pero se encuentran rodeados de municipios con valores altos, poniendo en riesgo a estos municipios por su cercanía, dentro de ellos se encuentran San Pablo del Monte localizado en el Estado de Tlaxcala, seguido de Comondá en el Estado de Baja California, San Salvador Atenco, Xalatlaco, Ocoyoacac, Tianguistenco, San Mateo Atenco en el Estado de México, Cuautlancingo en el Estado de Puebla, Elota en el Estado de Sinaloa, Conkal en el Estado de Yucatán, entre oros.

Dentro de los municipios con una afectación baja se encuentran la Heroica Ciudad de Huajuapan de León, de Tlaxiaco, de Juchitán de Zaragoza, Asunción Nochixtlán en el Estado de Oaxaca, entre otros.

\section{CONCLUSIONES}

Se concluye que el comportamiento espacio temporal de la pandemia, es diferenciado en el tiempo y en el territorio, según las fases de desarrollo de la pandemia.

La primera fase se caracteriza por contagios importados concentrándose en la Cd. de México, estado de México y ciudades que cuentan con aeropuerto internacional.

Para la segunda fase, se inician los contagios comunitarios a ciudades cercanas a las antes señaladas haciendo difícil un seguimiento específico.

La tercera fase, se caracteriza por un crecimiento de casos en forma exponencial, que se registran en las ciudades con tamaño de población y densidades altas, por ejemplo la ciudad de México, Estado de México, Puebla, las ciudades fronterizas del norte, además de las ciudades de los estados de la vertiente del Golfo de México.

Las políticas generales de contención de la pandemia incluye la cuarentena como medida principal, y la movilidad de la población juega un papel principal para cortar las cadenas de contagio, Sin embargo la mayor parte de los estados registra una disminución de movilidad por debajo del $50 \%$, por lo que se debe fortalecer esta medida.

Los métodos empleados para el análisis espacial, señalan que existen grupos o clusters de municipios que son focos rojos porque registran el mayor número de casos. En esta tercera etapa el contagio es local, por lo que alrededor de estos focos rojos se tienen que implementar medidas específicas más rigurosas, para disminuir el número de casos.

Para los grupos de municipios que tienen valores altos y sus vecinos registran valores bajos, es importante implementar medidas rigurosas a aquellos que tienen valores altos y proteger a los municipios de valores bajos, se trata de políticas diferenciadas.

Aquellos municipios con valores bajos y se encuentran acorralados por municipios con valores altos, se deben implementar políticas rigurosas al igual que los municipios vecinos.

En los municipios con valores bajos rodeados por municipios con valores bajos, conforman el potencial de población sana, en donde es importante no bajar la guardia, porque estamos a inicio de la tercera fase.

\section{REFERENCIAS}

Gobierno Federal. Secretaría de Salud (2020). Comunicado técnico diario nuevo coronavirus en el mundo (COVID-19). Disponible en: https://www.gob.mx/salud/documentos/informacion-internacionaly-nacional-sobre-nuevo-coronavirus-2019-ncov

INFOBAE, (2020) México es el quinto país más afectado por el COVID-19 en América y aún están por venir las semanas más críticas: OPS. Disponible en: 
https://www.infobae.com/america/mexico/2020/04/29/mexico-es-el-quinto-pais-mas-afectado-por-elcovid-19-en-america-y-aun-estan-por-venir-las-semanas-mas-criticas-ops/

Instituto Nacional de Estadística y Geografía 2010 Conteo de Población y vivienda, disponible en: https://www.inegi.org.mx/programas/ccpv/2010/

Instituto Nacional de Estadística y Geografía 2015 Encuesta intercensal Disponible en: https://www.inegi.org.mx/programas/intercensal/2015/

La Jornada (2020a) 22 de abril de 2020, autores: Néstor Jiménez y Alonso Urrutia

La Jornada (2020b) 28 de abril de 2020, autora: Ángeles Cruz Martínez.

La Jornada (2020c) 26 de abril de 2020, autora: Laura Poy Solano).

Mitchell, Andy. La Guía de Esri para el análisis SIG, Volumen 2. Esri Press, 2005.

OMS (2020) Coronavirus disease (COVID-2019) situation reports.26 e abril de 2020. Disponible en: https://www.who.int/docs/default-source/coronaviruse/situation-reports/20200426-sitrep-97-covid19.pdf?sfvrsn=d1c3e800_6

Santana G. y Aguilar A. G. 2019. Método Para Autocorrelación Espacial (Aplicación a la mortalidad por homicidios en el Estado de México) en Métodos cuantitativos en Geografía humana, Buzai G. Santana M. Argentina.

Santana M. (2020). COVID-19 en México: Comportamiento espacio temporal y condicionantes socioespaciales, febrero y marzo de 2020. Revista Posición 2020,3. Dossier: Análisis geográfico del COVID-19. ISSN 2683-8915 [en línea]. Recuperado de https://posicionrevista.wixsite.com/inigeo/numero-3-2020

Unwin D. 1996 "GIS, spatial analysis and spatial statistics" Progressin human Geography. California:SAGE Publications, 20, 4 p. 540-551. https://doi.org/10.1177/030913259602000408 